\title{
Measurements revealing Challenges in Radar Sensor Modeling for Virtual Validation of Autonomous Driving
}

\author{
Martin Holder, Philipp Rosenberger, \\ Hermann Winner \\ Technische Universität Darmstadt \\ Darmstadt, Germany \\ \{holder, rosenberger, winner\}@ @zd.tu-darmstadt.de
}

\author{
Thomas D'hondt \\ Siemens Industry Software NV \\ Leuven, Belgium \\ thomas.dhondt@siemens.com
}

\author{
Vamsi Prakash Makkapati, Michael Maier, \\ Helmut Schreiber, Zoltan Magosi \\ Graz University of Technology \\ Graz, Austria \\ \{vamsi.makkapati, f.m.maier, \\ helmut.schreiber, zoltan.magosi\} @ tugraz.at
}

\author{
Zora Slavik, Oliver Bringmann, Wolfgang Rosenstiel \\ FZI Research Center for Information Technology \\ Karlsruhe, Germany \\ \{slavik, bringman, rosen\}@fzi.de
}

\begin{abstract}
The virtual validation of automated driving functions requires meaningful simulation models of environment perception sensors such as radar, lidar, and cameras. There does not yet exist an unrivaled standard for perception sensor models, and radar especially lacks modeling approaches that consistently produce realistic results. In this paper, we present measurements that exemplify challenges in the development of meaningful radar sensor models. We highlight three major challenges: multi-path propagation, separability, and sensitivity of radar cross section to the aspect angle. We also review previous work addressing these challenges and suggest further research directions towards meaningful automotive radar simulation models.
\end{abstract}

Index Terms-Radar Sensor Model, Autonomous Driving, Virtual Validation

\section{INTRODUCTION}

Autonomous driving is seen as one of the key technologies that is influencing and shaping the future of mobility.It is mandatory that the automated driving function (ADF) reacts safely in all possible situations, thus requiring rigorous testing during its development. However, it is still unclear how ADFs may be tested in an efficient manner. Research projects such as ENABLE-S3 [1] and PEGASUS [2] aim to establish alternative testing methods for safety assurance of autonomous driving, such as validation in simulation environments. Virtual

This work has been conducted within the ENABLE-S3 project that has received funding from the ECSEL Joint Undertaking under grant agreement no 692455. This joint undertaking receives support from the European Union's HORIZON 2020 research and innovation program and Austria, Denmark, Germany, Finland, Czech Republic, Italy, Spain, Portugal, Poland, Ireland, Belgium, France, Netherlands, United Kingdom, Slovakia and Norway. Additionally Z. Slavik acknowledges the support of the University of Tuebingen with the graduate program EAES founded by the State of Baden-Wuerttemberg, Germany, Ministry of Science, Research and Arts. testing methods have the potential to accelerate the release of ADFs [3] and can be exploited during the entire development process. Verification and validation setups start from fully simulation-based approaches and should be able to gradually integrate more physical components from the final system. For ADFs, this implies either stimulating the environment perception sensors (i.e. over-the-air), or substituting real sensors with virtual sensor models. In both cases, the objective is to build simulation models within a virtual test architecture, whose behavior is indistinguishable, within generally accepted criteria, from the behavior in the real world. In this paper we focus on challenges in the development of radar sensor models to be used in Vehicle-, Software-, and Model-in-theLoop testbeds.Three major challenges are identified using real world measurement data. These challenges are known to be easy to observe, but difficult to model and include multi-path propagation, separability, and the sensitivity of radar cross section (RCS) to aspect angle.

The remainder of this paper is organized as follows: Section III gives a brief overview about radar sensors and their role in autonomous driving, while highlighting unresolved issues in state-of-the-art radar sensor modeling. The third chapter presents real-world radar measurements that exhibit challenging phenomena, such as multi-path reflection, separability, and sensitivity to RCS. We link our observations to modeling approaches and discuss their feasibility. We conclude by outlining remaining challenges and further research directions towards meaningful and trustworthy automotive radar simulations to enable virtual testing of ADFs. 


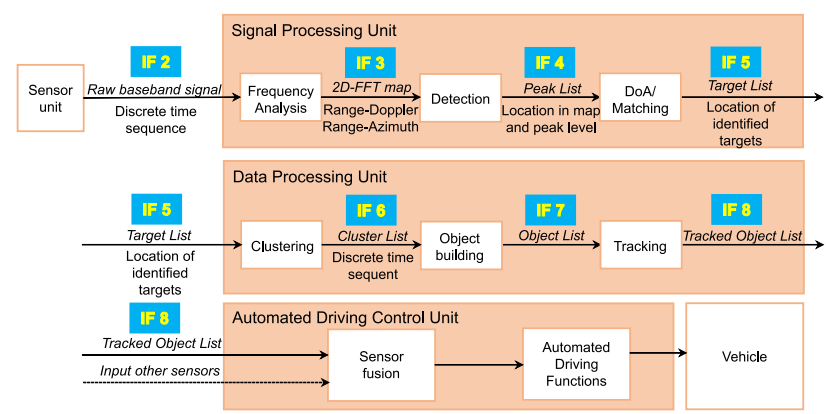

Fig. 1: Automotive radar signal and data processing flow

\section{Radar SEnsors in Autonomous Driving}

Early generations of driver assistance systems typically used a single environment perception sensor for each driver assistance function. Today's higher levels of automation often deploy sensor fusion concepts or semantic grids to detect also stationary objects [4]. The role of radar for driving automation has become increasingly important; with further developments towards higher resolution [5] and fully polarimetric devices [6], radar is considered a key sensor for autonomous driving [7]. Among the primary reasons for the success of radar are that it is more robust against adverse weather conditions compared to lidar or camera, and that it is able to measure the target's velocity via the Doppler-effect in addition to its range [8].

\section{A. Radar sensor fundamentals}

State-of-the-art automotive radars utilize frequency modulated continuous wave (FMCW) modulation with fast chirp sequences. Phased array antenna systems allow digital beamforming. For each detection, the radar sensor measures the distance $R$, azimuth and potentially elevation angle as well as the radial component of relative velocity between radar and target. The relative amount of reflected energy indicates the radar cross section (RCS) of the target. A generic radar processing chain is given in [9] and will be briefly reviewed here. We introduce an interface description (see Fig. 1) to clarify our notation of signals and data streams. After down conversion and sampling, the first (digital) signal available in a FMCW radar is the beat signal at IF2. A two-dimensional FFT produces the range and Doppler spectrum. In phasedarray antenna systems, a third FFT is applied to the antenna elements to obtain the azimuth angle to the target. Peaks in the spectra can be determined with CFAR methods and are then arranged in a peak list (IF4). After resolving ambiguities, possibly caused by aliasing effects and multi-path propagation, the actual location of the reflections can be displayed in a polar coordinate system along with the associated relative speed and power (IF5). It is also possible to combine individual reflections to produce larger targets (IF6). Object detection is often conducted by first proposing object hypotheses (IF7) and then applying an object tracking algorithm to the tracked object list (IF8). In an ADF, either a list of tracked objects, or less processed data is often fused into one environment model.

\section{B. State-of-the-art methods for modeling automotive radar}

Many approaches for radar sensor modeling have been reported in the literature. Indeed, the right trade-off between simulation realism, parametrization complexity, and computational speed must be found [10]. Therefore, a broad range of simulation models have been studied, each focusing on different applications and fidelity requirements. Historically, the first sensor models studied are often simplified abstract models, also called ground-truth or object list sensor models. Those models filter ground-truth information directly available in the environment simulation and emulate idealized behavior of the radar sensor. Such models do not exhibit errors in detection and measurement of object states, and output ideal simulation values in the format of a radar object-list [11]. Even though those models typically lack most of the sensor characteristics observed in real measurements, they are easy to parameterize and fast to execute. This makes them useful in early design phases in order to validate the functional behavior of an ADF in either ideal conditions or under the assumption that no sensor errors must be considered.

Phenomenological models offer an intermediate level of simulation fidelity. They try to emulate the physical behavior of the radar through the usage of statistical laws and simplified physical equations or maps, which are applied to groundtruth simulation data. Therefore, they can be seen as an extension of the ground-truth radar sensor models, but include typical sensor characteristics such as measurement noise, clutter detections, the variation of the detection amplitude as a function of the target position and orientation, and limited resolution [12]. They offer some physical insights into the behavior of the system, while being reasonable to parameterize and sufficiently efficient to execute in realtime or faster than real-time. Sensor models based on ray tracing techniques simulate the propagation of electromagnetic waves in the environment using asymptotic approximations, such as geometrical or physical optics [13], [14]. These models can simulate additional physical phenomena such as multi-path propagation, ghost targets, and interference, which can lead to errors in environment perception [15], [16]. However, such methods have several limitations. First, they are computationally expensive, limiting their use in real-time applications. Speed of execution is a limitation when considering the sheer amount of scenarios that must be simulated in order to validate an ADF. It should be noted that recent developments in ray tracing are gradually reducing this limitation [16]. Second, the environment model requires a high level of detail, particularly in the geometry and material properties of all static and dynamic objects. This detail is difficult and expensive to measure. Third, to obtain an object list, ray tracing models must execute all procedures in the radar pipeline, which for commercial sensors are typically proprietary and thus not accessible. 
Data driven approaches try to bypass these requirements on model and environment parametrization by using black-box sensor models, which approximate radar outputs from realworld sensor readings. For instance, previous work has studied the computation of a joint probability distribution linking the ground-truth position of a target to the corresponding measured sensor output [17]. Sensor-specific characteristics can hereby be learned directly from real-world data, as long as the data set is representative of those phenomena. Furthermore, black-box sensor models do not require computationally expensive ray tracing techniques. An approach using variational auto-encoders for estimating the radar power field was also investigated [18].

Finally, work executed for simulation environments [19] and real-world measurements [20] shows that echoes of a typical passenger car can be summarized by a set of characteristic scattering points, which result in an equivalent but simplified object representation. Therefore, scattering center approaches try to offload the complex computations required to compute this reduced set of reflectors offline and use the simplified object representation in order to enable fast and accurate online computations [21], [22]. This type of model is difficult to scale, as it forces the user to compute equivalent models for every type of object in the environment. Additionally, equivalent models of static targets such as trees or sign gantries have yet to be developed. Ultimately, common road objects, such as guardrails, bridges, and tunnels, which are known to have a large impact on wave propagation behavior, are not covered by these models.

\section{Interim conclusion: Why is the problem of radar sensor modeling not yet (fully) solved?}

Despite the promising approaches listed above, we draw at this point the following interim conclusions on the stateof-the-art towards meaningful virtual test-drives:

Requirements and intended areas of applications: Requirements do not yet exist for a sensor model eligible for the virtual validation of ADFs. Unlike in a typical product development process, requirements are apparently stated by the modeler, rather than by the model's user. Therefore, most radar sensor models are not developed to the same requirements. Their eligibility for application in test architectures (HiL, MiL, SiL, ViL) remains unclear.

Test Cases: Across our literature review, we noticed a lack of test methods and systematic benchmarks that allow an unbiased comparison of the reported models. Also, the reported models often lack comparable validation test reports, leaves it unclear as to whether the requirements have been fulfilled. From this observation we conclude that no ultimate statement regarding the reliability of a sensor simulation result may be drawn.

Model Validation: Although a large variety of radar sensor models exist, neither model templates, quality criteria, systematic tests, nor a method for validating the models' quality in terms of model fidelity exist. There is a lack of a measure for trust in simulation results, and in particular in situations that the model has not been explicitly trained on. Therefore, the prediction capability of a model in varied test architectures, as already mentioned, cannot be guaranteed. This fundamentally limits the explanatory power of simulation results.

Modeling Method: We have observed that the vast majority of approaches use ray tracing to mimicking advanced wave propagation phenomena. However, no approach systematically discusses the fundamental limitations of ray tracing for its application in automotive radar sensor models. In particular, no in-depth study of the fidelity of radar-specific bidirectional reflectance distribution functions (BRDFs), the essential part of the rendering process, has been reported.

Model Integration and Model Exchange: Radar sensor models cannot be universally applied, as they are tightly coupled to their environment representation. Consequently, the accuracy of the sensor model's output is limited by the environment simulation. While simulation environments that exploit photo-realistic rendering techniques tend to accurately represent visual sensors, they tend to produce poor inputs for radar models regarding object materials, thicknesses and surfaces.

\section{TACKLING CHALlENGES FOR RADAR SENSOR MODELS}

Many experiments were carried out under the ENABLE-S3 project to test and derive validation criteria for radar sensor models. The goal of these experiments, conducted at the Griesheim August-Euler-Airfield, was to establish a database for sensor modeling and validation, including specific scenarios that focus on radar-specific phenomena. Multiple cars, equipped with different radar and lidar sensors, a motorbike, a trailer, and a truck where incorporated. Agent positions were obtained with DGPS measurement devices. This section analyzes these experiments for phenomena such as occlusion, separability, and sensitivity of RCS to aspect angle.

\section{A. Experiment 1: Occlusion}

It is well known that radar sensors are, due to multi-path effects, technically able to detect targets that are not within line of sight (LoS). In the studied scenario, two cars are driving in the same lane in front of the ego car, causing the front-most car to be occluded (i.e. no direct LoS). An adaptive cruise control system maintains a constant time-gap between the vehicles as they drive at approx. $60 \mathrm{~km} / \mathrm{h}$, see Fig. 2 The non-occluded car and the occluded car are $30 \mathrm{~m}$ and $60 \mathrm{~m}$ from the ego-car, respectively. RCS values were reported by a tracking algorithm running on the radar sensor in the ego car, which takes individual radar reflection points as an input to build an object track based on observations over time. The algorithm also reports the number of reflections associated with objects at each time-step. With this experiment, we are examining how occlusion influences the measured RCS and the number of reflection points. The RCS of the non-occluded car was found to have a mean value of $18.5 \mathrm{dBsm}$ over three repetitions. The measured RCS and number of reflection 
points associated to each object is given in Fig. 3 . Occlusions cause the RCS to drop from $18.5 \mathrm{dBsm}$ to $10.6 \mathrm{dBsm}$ (see Fig. 3a. The number of reflection points varies stochastically when occlusion occurs (see Fig. 3b), which contradicts a possible linear relation between distance and the number of reflection points. While in the non-occluded case, the car causes up to 6 reflections, up to 4 reflections are reported in the occluded situation.

\section{B. Modeling occlusion with ray tracing}

In a sensor model, wave propagation must be designed in a manner that allows for efficient calculation of multipath reflections. Although ray tracing has been repeatedly suggested for automotive radar simulation [14], no study measures the performance of modeling multi-path effects by comparing to real world measurements. In particular, the varying number of reflections points is challenging for ray tracing approaches as valid paths are inherently coupled with the number of emitted rays. In the RASIG software package [16], [23], a ray tracer along with Phong BRDF [16] is utilized to compute the energy reflected from each hit-point. The Phong BRDF does not impose any special requirements on the resolution of the input 3D geometries. Rays are generated according to the measuring range of the sensor and each ray is propagated through the scene until it hits a surface or is terminated. At any hit-point, the reflected energy is computed and a bouncing ray is shot if the maximum ray depth (i.e. predetermined number of reflections) has not yet been exceeded. Fig. 4 shows the result of RASIG for the scene used in experiment 1 . The top halve of Fig. 4 depicts the back-scattered energy in the radar field of view. The shade of pixels is proportional to the back-scattered energy. Energy from Multi-path hits is shown by coloring pixels, a red hue is added upon the first reflection, and a green hue upon the second. Thus, a yellow pixel corresponds to a ray that has struck a surface both after the first and second reflections. A ray that does not hit any objects is terminated and its pixel is set to black. A range-azimuth plot is given in the lower half of the figure (with range- and azimuth resolution of $0.2 \mathrm{~m}$ and $0.1^{\circ}$ respectively), which shows the range-azimuth bins colored in the same manner as described before. The simulation runs at $20 \mathrm{fps}$, with a total number of 170000 rays is cast into the scene. Modern radar has a typical update period of $50 \mathrm{~ms}$, and is used as a baseline for real-time capability. The number of rays striking the occluded car, the non-occluded car, and the pavement at different ray

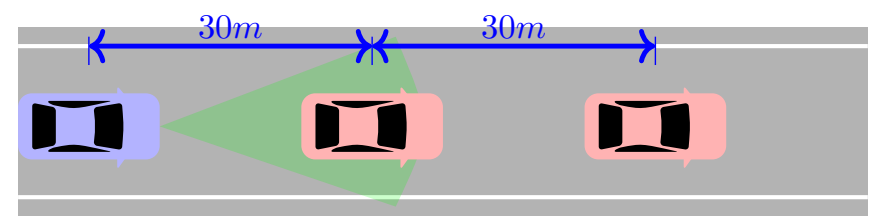

Fig. 2: Two cars driving in front of the ego-vehicle and spaced by $30 \mathrm{~m}$.

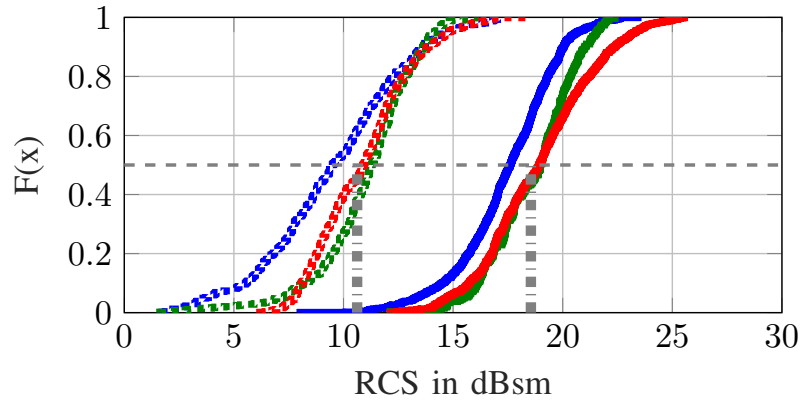

(a) Distribution of RCS for the studied car.

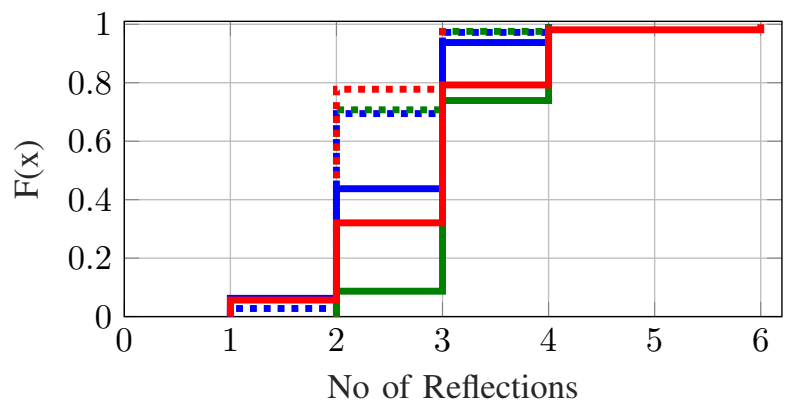

(b) Number of reflections points for studied car.

Fig. 3: Empirical CDF for RCS and number of reflection points. Solid line indicates non-occluded situation, while dashed line indicates occluded situation.

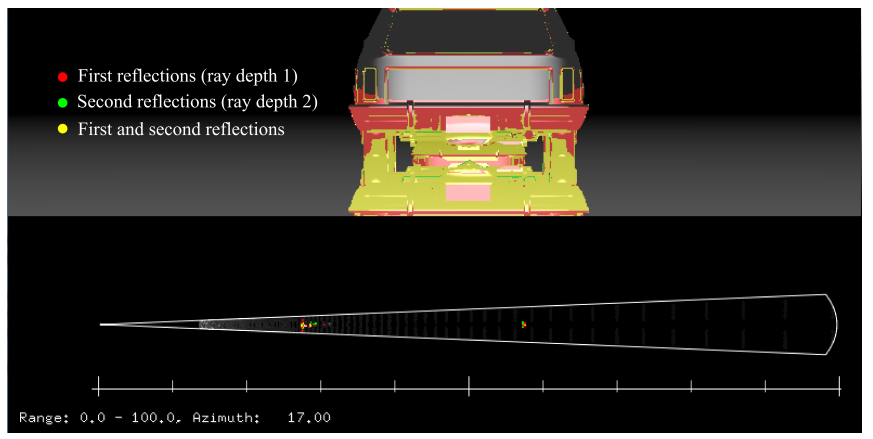

Fig. 4: Ray tracing based simulation showing the effect of multi-path reflections. Red, green and yellow indicates first, second, and third reflection respectively.

TABLE I: Number of rays hitting the cars at each ray depth

\begin{tabular}{|c|c|c|c|}
\hline & Ray depth: 0 & Ray depth: 1 & Ray depth: 2 \\
\hline occluded car & 0 & 1966 & 2167 \\
\hline non-occluded car & 26082 & 40088 & 45500 \\
\hline pavement & 82960 & 90668 & 98837 \\
\hline
\end{tabular}

depths are reported in Table I Notably, less than $5 \%$ of the rays reach the occluded car. As can be seen in Fig. 4, the occluded car is only visible due to multi-path propagation. It must be noted that the computational time required for ray tracing depends on many factors, including the number of rays, ray depth, and the amount of buffer size associated with 
each ray. Our results imply, however, that occlusion effects can conceptually be modeled by ray tracing, but the stochastic character of the number of reflection points is not addressed by this setup. Also, the number of hit points may initially not be seen as an equivalent measure for received power or allowing to infer about RCS of targets.

\section{Experiment 2: Separability}

The problem regarding separability in radar consists of two tasks: the capability of receiving and detecting separate peaks for objects that are located close to each other in azimuth, range rate, and range and the assignment of peaks to objects. The experiment placed several vehicles as close as possible to each other on a circular arc with radius $25 \mathrm{~m}$ while a radar-equipped vehicle approached these vehicles as depicted in Fig. 5d. The frequency ramp of the radar used in this experiment covered a bandwidth of $2 \mathrm{GHz}$ on the carrier frequency $77 \mathrm{GHz}$. The radar output was the beat signal (IF2) and signal processing was performed on a host PC. As a result, range azimuth maps indicate the location of normalized power levels in $\mathrm{dB}$ depending on range and azimuth direction. In Fig. 5 the color indicates the power level normalized to the maximum received power level of each snapshot. The $y$-axis depicts the range direction and the $x$ axis the azimuth direction. Measurement results are depicted in the range-azimuth maps for $10.8 \mathrm{~m}, 14.6 \mathrm{~m}$ and $17.2 \mathrm{~m}$ in Fig. 5. The peak levels of the two visible vehicles at $10.8 \mathrm{~m}$ are approximately equally strong, indicating the existence of at least two separate objects. At $14.6 \mathrm{~m}$ and $17.2 \mathrm{~m}$, the range of the vehicles relative to the radar comes into play. The radar is unable to resolve individual targets as all cars are within same range and range rate. Consequently, only one target gets resolved, while the others are obscured due to the strong reflection from the trailer.

Separability appears to be a good performance metric for generic radar models. Separability is well-described by the cell-volume concept, originally used to explain how physical constraints determine sensor performance [24]. The measurable space, called the cell-volume, is determined by the measurable range in each dimension (i.e. range, radial velocity, and azimuth angle). Here, a discretized 3-D volume, or cell, is defined by a bin in each dimension. An ideal point object (i.e. a point target) can be represented by a cell and a corresponding power return. The cell-volume concept utilizes the fact that detections are separable if there is at least one free bin in the adjacent range, range rate, or azimuth axes. One version of the cell-volume concept has already been successfully demonstrated for radar sensor modeling [25].

\section{Experiment 3: Variation of RCS during dual lane-change}

In the third experiment, the sensitivity of a car's RCS with respect to its heading and azimuth angle, denoted as $\Psi$ and $\phi$ respectively, in a real-world driving scenario is studied. To make sensitivity of RCS to small variations in azimuth and distance visible, we intentionally have chosen to use human drivers to obtain a natural variation in maneuvers. In our experiment, a car repeatedly performs dual lane changes while observed by the ego car's radar. A cruise control system in each car maintains $30 \mathrm{~km} / \mathrm{h}$ with a radial distance of $15 \mathrm{~m}$ between the ego and the target car throughout the experiment. The experiment setup is illustrated in Fig. 6 The reference RCS of the target car, $14.5 \mathrm{dBsm}$, was obtained by driving at a constant speed with both vehicles driving in a straight line. The measured RCS of the target car over heading and azimuth angle is depicted in Fig. 7, where colors decode deviation from the reference RCS. A few trials were selected and highlight exemplary that although trials are repeated in a similar manner, considerable deviations in RCS can be noticed and general tendencies are difficult to identify. While both the road surface (roughness profile and inclination) as well as the environmental condition were subjectively constant during the experiment, a slight variation in target distance and aspect angle is apparently sufficient for large deviations in RCS. To capture such behavior realistically by a sensor model, a highly detailed representation of every object in the scene is necessary. High requirements on the surface resolutions can be derived from the wavelength of an automotive radar usually operating at $77 \mathrm{GHz}$. For example, the preservation of phase information within $10^{\circ}$ requires that all surface elements are known with a depth resolution of $0.1 \mathrm{~mm}$ on any object visible to the radar in the virtual scene. Depending on the curvature, the surface elements must be meshed with sub- $\mathrm{mm}^{2}$ accuracy. Although scattering-center models as a replacement for such highly detailed models have been suggested, they usually do not consider the sensor mounting position or the distance behavior. Further environmental aspects (such as weather) or road surface conditions, which have a proven impact on interference phenomena, are also not modeled. Moreover, these models are usually designed exclusively for non-occluded car and do not apply to occluded vehicles, as was discussed in the occlusion experiment.

\section{Implications For a Radar Sensor Model}

The experiments above have shown major characteristic phenomena of an automotive radar sensor. Any radar sensor model should be able to recreate these characteristic phenomena. As an example we have shown the ray tracing based approach which models the multi-path propagation and thus in principle can detect vehicles that are not in the direct line of sight. The seperability issue as presented in Section III-C has implications for the radar sensor model. Within the model, the separability capabilities of the radar sensor must be represented correctly and thus, the information detail generated within the the model must be limited to the separability capabilities of the radar sensor. The cell-volume concept is a promising approach to address the separability problem, as it only requires a minimal set of radar parameters, most of which can be obtained from sensor datasheets. The issue of the variation of RCS over azimuth angle can be easily observed in measurements, but is not adequately captured by existing radar models. Many factors influence this behavior, including interference effects, making it difficult to allow an 


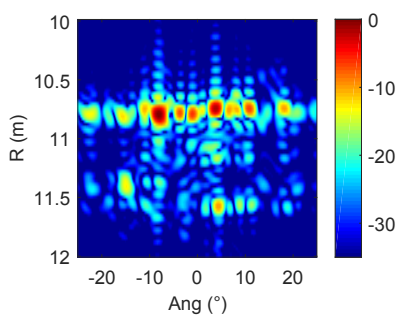

(a) Distance to $\operatorname{arc} d=10.8 \mathrm{~m}$. Separation of targets is possible.

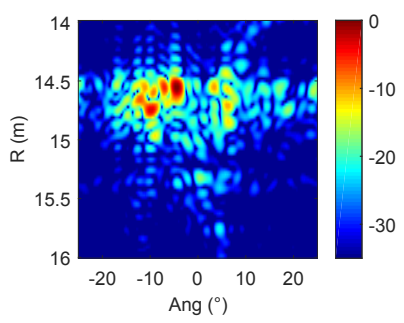

(b) Distance to arc $d=14.6 \mathrm{~m}$. Separation of individual targets is not possible.

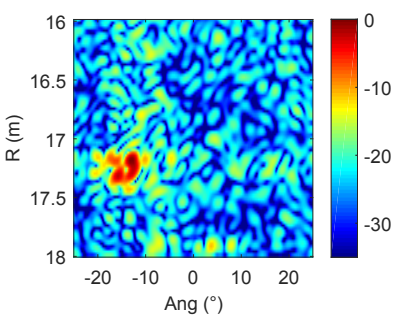

(c) Distance to $\operatorname{arc} d=17.2 \mathrm{~m}$. Separation of individual targets is not possible.

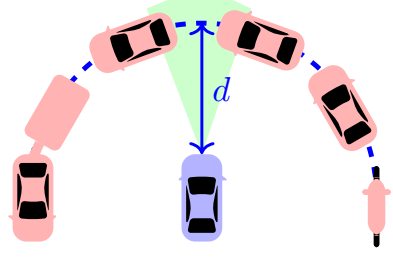

(d) Experiment design.

Fig. 5: Separability of targets on range-azimuth level at distances.

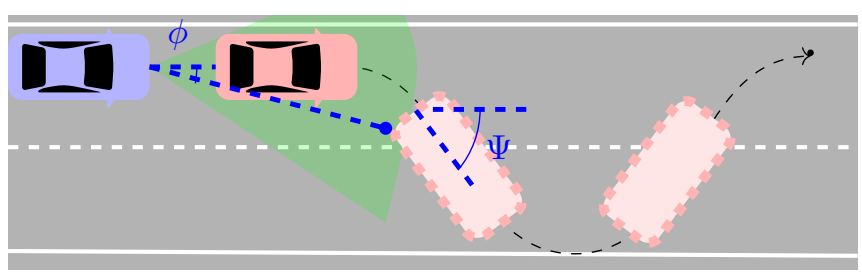

Fig. 6: Dual lane changing maneuver of the target (red) used to study the RCS distribution seen by the ego-vehicle (blue)

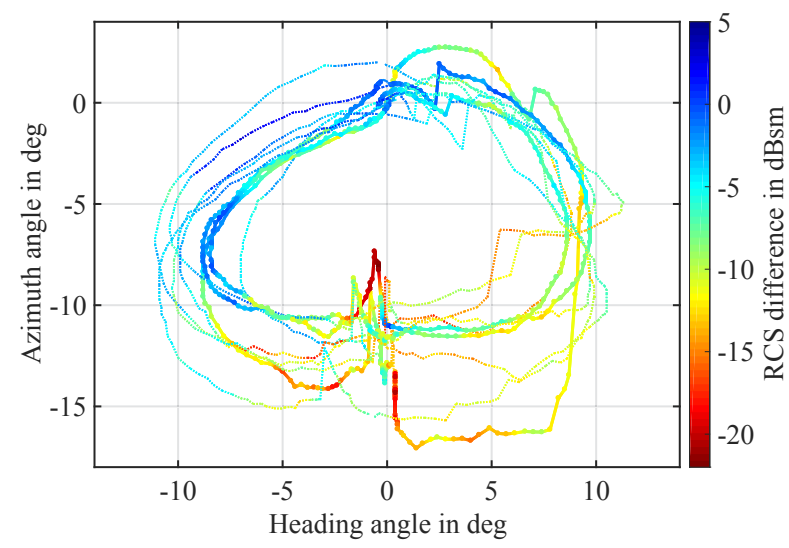

Fig. 7: Heading angle $(\Psi)$ vs. Azimuth angle $(\phi)$ in dual lane-change scenario. Figure reads counter-clockwise starting from origin.

efficient representation of environmental objects in models. A systematic analysis of any potential influencing factors on radar measurement is needed for the design and validation of a radar sensor model. Such an analysis is unfeasible using only real-world experiments. As we have shown, radar sensor measurements are characterized by their stochastic behavior, which complicates reproducibility, and often requires deep access into the sensor hardware to make effects fully visible. Such behavior complicates sensor model validation, as the singular-comparison of measurement to simulation results is not meaningful. Ray tracing based approach (being geometry based) is in principle able to simulate RCS variations due to changes in relative orientation between the vehicles (as in Section [III-D). But as mentioned earlier this behavior is influenced by many other factor as well making further research on radar-specific formulations for the ray tracing approach unavoidable. The necessary degree of fidelity in radar sensor models for the virtual testing of ADFs is not clear yet. Preliminary approaches for solving this issue have been released under the umbrella term purpose driven fidelity [26] and have already been deployed for object detection with lidar [27].

\section{A. Modular Sensor Model Architecture and Interfaces}

From our experiments, we conclude that despite its complexity, a modular simulation architecture of a radar sensor can be derived, where individual parts are (almost) independent. By subdividing a radar sensor model into environment simulation, wave-propagation and interaction, sensor hardware model and detection and tracking model, we aim to separate influencing factors while keeping modules interchangeable. This separation increases the flexibility for the individual design of model components, the adaption of individual sensors, and makes it easier to validate and verify sensor models. For example, radar phenomena, such as occlusion, are clearly linked to wave-propagation, while target separability depends on the underlying sensor hardware rather.

\section{B. Validation Experiments}

The advantage of a simulation environment, namely access to any internal parameters, comes with the burden of validating the measurements, taken as reference for simulation, itself. As indicated in our result, a ray tracer reports the exact location of a hit-point, while the radar wave does not. Tracking algorithms are technically able to report a number of associated reflections to each object, but this only allows for a limited statement regarding the total number of reflection points on each object, as the underlying tracking algorithm and its handling of ambiguous reflections is typically unknown. Also, a large number of effects are most clearly visible only under certain conditions. As demonstrated in the separability experiment, limitations in separation capability are easily visible on the baseband signal, but in the case of briefly vanishing detections, tracking algorithms are usually able to detect temporally invisible objects. The transferability of measurements results is limited by the large variety of peculiarities in sensor hardware, 
as the mounting position and the radar's antenna pattern play a major role in its detection capability.

\section{CONCLUSION}

This work has structured open issues in radar sensor modeling, which are obstacles that must be overcome on the path to meaningful virtual validation of ADFs. A number of research questions linked to realistic radar sensor models are identified: We briefly reviewed existing radar sensor models and highlighted unresolved issues towards meaningful radar sensor models, which are often linked to the question of model fidelity. Here, we particularly noticed that neither generally accepted requirements, nor validation criteria for radar sensor models, have been reported for virtual testing of ADFs. Therefore, it remains unclear to what extent radar sensor models can presently be utilized for meaningful autonomous driving simulations. Trust in simulation result requires metrics for sensor fidelity across phenomena.

We presented major radar specific phenomena - multipath propagation, seperability, and variation of RCS over azimuth angles - and discussed their significance for radar sensor modeling. We emphasized the need for a more specific design of experiments for radar sensor model validation. We also discussed the issue of sensor model validation and measurement data validation, which is complicated by the fact that radar measurements are characterized by highly stochastic variation and high sensitivity to small changes in influencing factors. Future work will investigate modeling methods for the efficient representation of a radar-specific environment and establish statistical verification and validation methods that account for the stochastic nature of radar sensor measurements.

\section{REFERENCES}

[1] European Initiative to Enable Validation for Highly Automated Safe and Secure Systems. [Online]. Available: https://www.enable-s3.eu

[2] Pegasus Research Project - Securing Automated Driving Effectively. [Online]. Available: http://www.pegasus-projekt.info/en/

[3] S. Sovani, "Simulation Accelerates Development of Autonomous Driving," ATZ worldwide, vol. 119, no. 9, pp. 24-29, 2017.

[4] J. Lombacher, K. Laudt, M. Hahn, J. Dickmann, and C. Wohler, "Semantic radar grids," in 2017 IEEE Intelligent Vehicles Symposium (IV). IEEE, 2017, pp. 1170-1175.

[5] F. Meinl, M. Stolz, M. Kunert, and H. Blume, "An experimental high performance radar system for highly automated driving," in 2017 IEEE MTT-S International Conference on Microwaves for Intelligent Mobility (ICMIM). IEEE, 2017, pp. 71-74.

[6] T. Visentin, J. Hasch, and T. Zwick, "Calibration of a fully polarimetric $8 \times 8$ Mimo FMCW radar system at $77 \mathrm{ghz}$," in 2017 11th European Conference on Antennas and Propagation (EUCAP). IEEE, 2017, pp 2530-2534.

[7] J. Dickmann, J. Klappstein, M. Hahn et al., "Automotive radar the key technology for autonomous driving: From detection and ranging to environmental understanding," in 2016 IEEE Radar Conference (RadarConf), IEEE, Ed. Piscataway, NJ: IEEE, 2016, pp. 1-6.

[8] A. Arage, W. M. Steffens, G. Kuehnle, and R. Jakoby, "Effects of water and ice layer on automotive radar," in Proc. of the German Microwave Conf, 2006.

[9] S. M. Patole, M. Torlak, D. Wang, and M. Ali, "Automotive Radars: A review of signal processing techniques," IEEE Signal Processing Magazine, vol. 34, no. 2, pp. 22-35, 2017.

[10] P. Cao, W. Wachenfeld, and H. Winner, "Perception sensor modeling for virtual validation of automated driving," it - Information Technology, vol. 57, no. 4, pp. 243-251, 2015.
[11] E. Roth, T. J. Dirndorfer, A. Knoll et al., "Analysis and Validation of Perception Sensor Models in an Integrated Vehicle and Environment Simulation," in Proceedings of the 22nd Enhanced Safety of Vehicles Conference (ESV 2011). Red Hook, NY: Curran Associates Inc, 2011.

[12] S. Bernsteiner, Z. Magosi, D. Lindvai-Soos et al., "Radar Sensor Model for the Virtual Development Process," ATZelektronik worldwide, vol. 10, no. 2, pp. 46-52, 2015.

[13] H. Ling, R. C. Chou, and S. W. Lee, "Shooting and bouncing rays: calculating the RCS of an arbitrarily shaped cavity," IEEE Transactions on Antennas and Propagation, vol. 37, no. 2, pp. 194-205, Feb 1989.

[14] G. Herz, B. Schick, R. Hettel, and H. Meinel, "Sophisticated sensor model framework providing real sophisticated sensor model framework providing realistic radar sensor behavior in virtual environments," 10th Graz Symposium Virtual Vehicle (GSVF), Graz, Austria, 2017.

[15] N. Hirsenkorn, P. Subkowski, T. Hanke et al., "A ray launching approach for modeling an fmcw radar system," 2017 18th International Radar Symposium (IRS), 2017.

[16] M. Maier, V. P. Makkapati, and M. Horn, "Adapting Phong into a Simulation for Stimulation of Automotive Radar Sensors," in International Conference on Microwaves for Intelligent Mobility, Munich, Germany, 2018.

[17] N. Hirsenkorn, T. Hanke, A. Rauch, B. Dehlink, R. Rasshofer, and E. Biebl, "Virtual sensor models for real-time applications," Advances in Radio Science, vol. 14, p. 31-37, 2016.

[18] T. A. Wheeler, M. Holder, H. Winner, and M. J. Kochenderfer, "Deep stochastic radar models," 2017 IEEE Intelligent Vehicles Symposium (IV), 2017.

[19] K. Schuler, D. Becker, and W. Wiesbeck, "Extraction of virtual scattering centers of vehicles by ray-tracing simulations," IEEE Transactions on Antennas and Propagation, vol. 56, no. 11, p. 3543-3551, 2008.

[20] M. Andres, P. Feil, W. Menzel, H. L. Bloecher, and J. Dickmann, "3D Detection of Automobile Scattering Centers Using UWB Radar Sensors at 24/77 GHz," IEEE Aerospace and Electronic Systems Magazine, vol. 28, no. 3, pp. 20-25, 032013.

[21] M. Bühren and B. Yang, "Simulation of automotive radar target lists using a novel approach of object representation," IEEE Intelligent Vehicles Symposium, 2006.

[22] H. Buddendick, T. Eibert, and J. Hasch, "Bistatic scattering center models for the simulation of wave propagation in automotive radar systems," in German Microwave Conference Digest of Papers, 2010, pp. 288-291.

[23] M. Maier, V. P. Makkapati, and M. Horn, "Environment Perception Simulation for Radar Stimulation in Automated Driving Function Testing," e \& i Elektrotechnik und Informationstechnik, in press.

[24] H. Winner, "Automotive RADAR," in Handbook of driver assistance systems, H. Winner, S. Hakuli, F. Lotz, and C. Singer, Eds. Cham: Springer Reference, 2016, pp. 325-403.

[25] P. Cao, "Modeling active perception sensors for real-time virtual validation of automated driving systems," Ph.D. dissertation, Technische Universität, Darmstadt, 2018.

[26] E. Drenth, "Virtual Vehicle Architecture (VVA)," SVEA - Vehicle Dynamics seminar, 06.07.2016.

[27] M. Holder, P. Rosenberger, F. Bert, and H. Winner, "Data-driven derivation of requirements for a lidar sensor model," 11th Graz Symposium Virtual Vehicle (GSVF), Graz, Austria, 2018. 